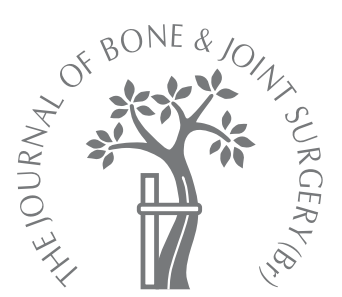

R. Niimi,

A. Sudo,

M. Hasegawa,

A. Fukuda,

A. Uchida

From the Department of Orthopaedic

Surgery, Mie

University Graduate School of Medicine, Mie, Japan

R. Niimi, MD, Research

Fellow

A. Sudo, MD, PhD, Assistant

Professor

M. Masegawa, MD, PhD,

Assistant Professor

A. Fukuda, MD, PhD,

Orthopaedic Surgeon

A. Uchida, MD, PhD,

Professor, Chairman

Department of Orthopaedic

Surgery

Mie University Graduate

School of Medicine, 2-174

Edobashi, Tsu City, Mie 514-

8507, Japan.

Correspondence should be sent to $\operatorname{Dr}$ A. Sudo; e-mail:

a-sudou@

clin.medic.mie-u.ac.jp

(C)2006 British Editorial Society of Bone and Joint Surgery doi:10.1302/0301-620X.88B11. $18063 \$ 2.00$

J Bone Joint Surg $[\mathrm{Br}]$ 2006;88-B:1438-40.

Received 2 May 2006; Accepted 26 July 2006

\title{
Changes in bone mineral density in transient osteoporosis of the hip
}

Transient osteoporosis of the hip is a disorder characterised by pain, and associated with temporary osteopaenia. Although osteopaenia is the essence of the condition, data do not exist about the local bone density of the femoral neck if no medication is administered. We describe three patients who were treated with limitation of weight-bearing only. Repeated bone mineral density measurements were obtained, and that at the femoral neck was lowest two months after the onset of the condition. The mean reduction in bone mineral density when compared with an age-matched control group was $13 \%$ (3\% to $24 \%)$. Spontaneous recovery was observed in all patients.

Transient osteoporosis of the hip is a rare condition of unknown aetiology, characterised by increasing hip pain and functional disability. Symptoms increase gradually over one to two months. Radiologically, loss of radiodensity becomes apparent within two months. Generally, the symptoms subside spontaneously and radiological findings return to normal, a few months later. ${ }^{1-4}$ Loss of bone mineral density (BMD) in the proximal femur is usual in this condition, however repeated measurements of BMD in patients who have had no drug treatment for the condition have not been previously reported. We report the results of three patients with transient osteoporosis of the hip who were treated with limitation of weightbearing only.

\section{Patients and Methods}

The medical records, BMD measurements, radiographs and MRI scans of three hips from three patients with transient osteoporosis of the hip were reviewed. All patients underwent dual energy X-ray absorptiometry (DEXA; Lunar DPX-L, Madison, Wisconsin), and repeated measurements of BMD were taken from both hips. Values obtained were compared with healthy subjects matched for age and gender. None of the patients had previously suffered a fracture of the hip, nor did they possess risk factors for avascular necrosis of the femoral head, or a family history of osteoporosis. No therapeutic intervention other than continued non-weight-bearing was attempted. We diagnosed transient osteoporosis of the hip as radiological evidence of a focal loss of radiodensity in the femoral head, pain in the hip for between two and six months, and MRI evidence of bone marrow oedema.

\section{Results}

Case 1. A 46-year-old man presented with a two-month history of pain in the right hip. He had suffered transient osteoporosis of the left hip eight years earlier, and had been treated successfully with conservative management. He had a limp and limited movement of the affected hip.

Plain radiographs showed demineralisation of the femoral head, neck and intertrochanteric area, without narrowing of the joint space (Fig. 1). An MR scan showed bone marrow oedema with decreased signal intensity in the femoral head, neck and intertrochanteric area on T1-weighted images (Fig. 2a), and increased signal intensity of the same areas on T2weighted images (Fig. 2b). The patient was given no medication and was not permitted to weight-bear. Four months after the onset of symptoms he was asymptomatic. The patient began weight-bearing at five months, and an MR scan performed at seven months showed normal signal intensity on both T1- and T2weighted images.

The BMD measurements at presentation, two months after the onset of symptoms, showed the right femoral neck to be $13 \%$ lower than the mean BMD of age-matched controls, and $31 \%$ lower than the BMD in the left femoral neck (Table I). The BMD of the lumbar spine was normal. At 12 months the $\mathrm{BMD}$ of the right femoral neck was similar to 


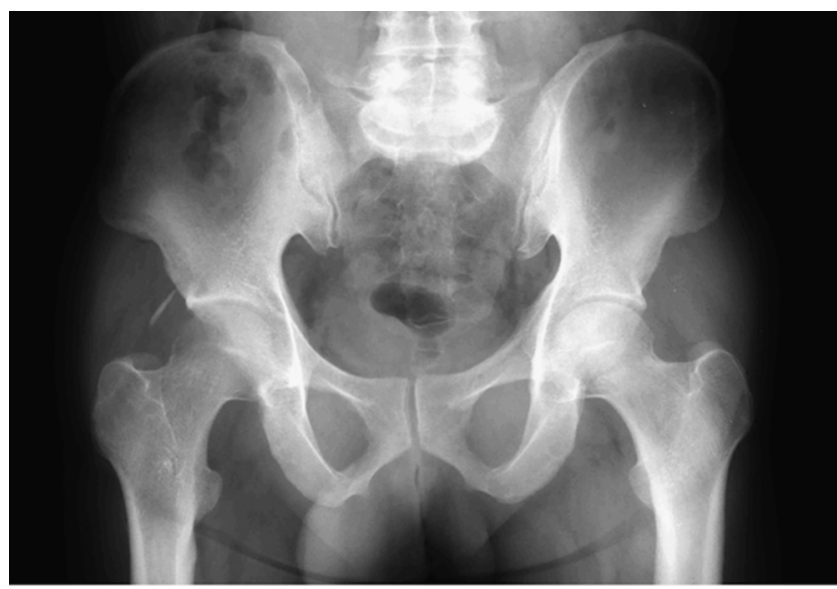

Fig. 1

Anteroposterior radiograph of the pelvis showing decreased radiodensity in the right femoral head, with a well-preserved joint space.

that of the left. The BMD in the right femoral neck was $2 \%$ higher than the mean BMD of the age-matched controls. The BMD of the right femoral neck showed its minimum value two months after the onset of symptoms.

Case 2. A 40-year-old man presented with a one-month history of pain in the left hip and a limp. Plain radiographs showed demineralisation of the left femoral head, neck and the intertrochanteric area with normal findings of the right femur. An MR scan revealed bone marrow oedema. Nonweight-bearing was initiated, and pain resolved three months later.

Measurements of BMD obtained two months after the onset of symptoms showed that in the left femoral neck it was $24 \%$ below the mean BMD of age-matched controls (Table II). In the right femoral neck the BMD was $6 \%$ below the mean BMD of age-matched controls. Seven months after the onset of symptoms, the BMD of the left femoral neck had improved. The BMD of the left femoral neck showed its minimum value two months after the onset of symptoms.

Case 3. A 60-year-old man presented with progressive pain in the left hip and an inability to bear weight. There was no history of trauma. Plain radiographs showed demineralisa-

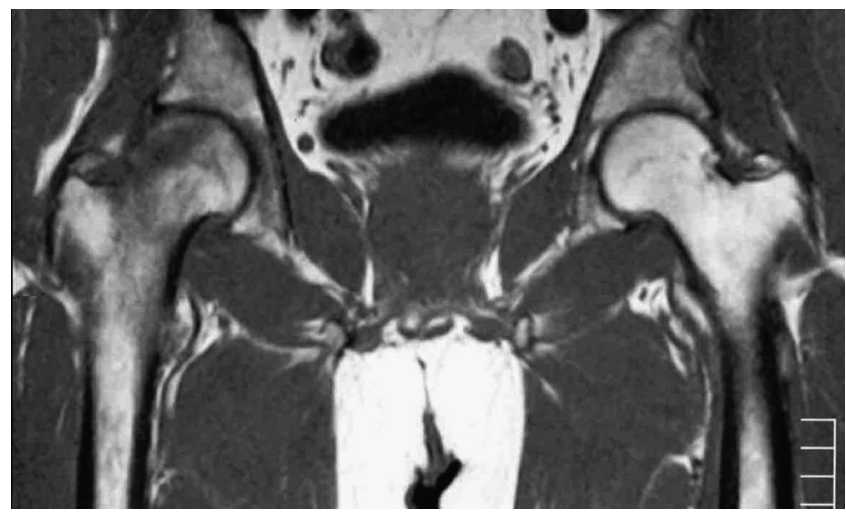

Fig. 2a

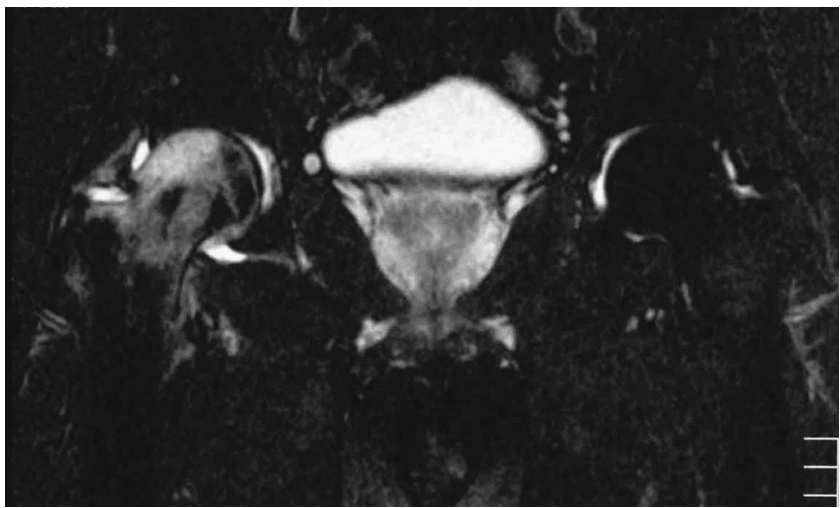

Fig. 2b

MRI of the hips showing a) diffusely decreased signal intensity in the right femoral head and neck on T1-weighted images and b) increased signal intensity in the same areas on T2-weighted images.

tion of the left femoral head. An MR scan revealed bone marrow oedema. The patient was advised against weightbearing and was given crutches. The pain had gradually decreased by three months and the patient was asymptomatic at four months. Two months after the onset of symptoms, the BMD in the left femoral neck was reduced to $3 \%$ lower than the mean BMD of age-matched controls (Table III). The BMD had increased to $3 \%$ higher than that of agematched controls at three months. Five months after the onset, an MR scan showed normal signal intensity. The

Table I. Repeated bone mineral density (BMD) measurements for case 1 (as a percentage of the BMD of age-matched controls $(100 \%)$

\begin{tabular}{lcrrrr}
\hline & \multicolumn{2}{l}{ BMD } & & \\
\cline { 2 - 6 } Months after onset of symptoms & \multicolumn{2}{l}{} & \multicolumn{1}{c}{$\mathbf{3}$} & \multicolumn{1}{c}{$\mathbf{5}$} & $\mathbf{1 2}$ \\
\hline Right femoral neck & 87 & 87 & 89 & 90 & 102 \\
Left femoral neck & 118 & 118 & 118 & 115 & 113 \\
\hline
\end{tabular}


Table II. Repeated bone mineral density (BMD) measurements of case 2 (as a percentage of the BMD of age-matched controls $(100 \%)$ )

\begin{tabular}{llll}
\hline & BMD & & \\
\cline { 2 - 4 } Months after onset of symptoms & $\mathbf{2}$ & $\mathbf{5}$ & $\mathbf{7}$ \\
\hline Right femoral neck & 94 & 97 & 97 \\
Left femoral neck & 76 & 91 & 95 \\
\hline
\end{tabular}

Table III. Repeated bone mineral density (BMD) measurements of case 3 (as a percentage of the BMD of age-matched controls $(100 \%)$ )

\begin{tabular}{llll}
\hline & \multicolumn{3}{l}{ BMD } \\
\cline { 2 - 4 } Months after onset of symptoms & $\mathbf{2}$ & $\mathbf{3}$ & $\mathbf{5}$ \\
\hline Right femoral neck & 106 & 111 & 114 \\
Left femoral neck & 97 & 103 & 118 \\
\hline
\end{tabular}

BMD of the left femoral neck showed its minimum value two months after the onset of symptoms.

\section{Discussion}

Transient osteoporosis of the hip is a self-limiting condition of unknown cause and was first described by Curtiss and Kincaid ${ }^{1}$ in 1959. Its clinical progression can be divided into three phases: 1) onset of pain and disability, 2) demineralisation of the femoral head, neck and trochanteric area without narrowing of the joint space and 3) resolution of symptoms and recovery of bone mineralisation. ${ }^{2}$ Demineralisation of the femoral head and neck has been reported to become apparent on plain radiographs one to two months after the clinical onset. ${ }^{1-3,5,6}$ In our study, a focal loss of radiodensity was apparent on plain radiographs at a mean of 1.3 months ( 1 to 2), and plain radiographs and MRI obtained at five to seven months showed complete recovery.

Several forms of treatment have been described including corticosteroids, ${ }^{7}$ bisphosphonates, ${ }^{5,8}$ and calcitonin. ${ }^{9}$ Carmona-Ortells et $\mathrm{al}^{7}$ reported two patients with transient osteoporosis of the hip treated with deflazacort. Complete recovery occurred between two and four weeks after initiating treatment. Montagna et $\mathrm{al}^{8}$ started treatment with an intramuscular amino-bisphosphonate (neridronate sodium, $25 \mathrm{mg} / \mathrm{month}$ ), calcium carbonate and cholecalciferol two months after the onset of symptoms and the patient became asymptomatic two months later (four months after the onset). Calcitonin has also been reported to help recovery within six to nine weeks. ${ }^{9}$ In our study the symptoms resolved at a mean of four months (3 to 5) without any medication, and drug therapy may therefore not be needed. Little has been reported about the serial changes in BMD. In 1996, Varenna et $\mathrm{al}^{3}$ reported three cases of transient osteoporosis of the hip in which the BMD of the proximal femur was evaluated 2.3 months after the onset of the disease. They found that the mean BMD of the femoral neck was $72.3 \%$ compared with the mean BMD of age-matched controls. However, all their patients were treated with intravenous clodronate. In 2002, Varenna et $\mathrm{al}^{5}$ evaluated BMD repeatedly using DEXA. At four months after the clinical onset, there was a mean increase of $12.3 \%$ (7.8\% to $26.9 \%$ ) in the BMD of the femoral neck. However, all their patients were treated with intravenous pamidronate. Changes of BMD in transient osteoporosis of the hip, but without drug therapy, have not been reported. In our study, the BMD at the femoral neck showed its minimum value two months after the onset of symptoms. The mean reduction in the $\mathrm{BMD}$ was $13 \%$, and spontaneous recovery was observed in all patients.

A pathological fracture is perhaps the most serious complication of transient osteoporosis of the hip. Among the elderly, low BMD is one of the major risk factors of fracture of the proximal femur. However, in transient osteoporosis of the hip, the affected patients are usually either healthy middle-aged men, or women in the third trimester of pregnancy. The BMD in these patients is usually normal prior to the onset of symptoms. Nguyen, Center and Eisman ${ }^{10}$ described three factors which can predict fracture risk: 1) the rate of bone loss of the femoral neck, 2) the baseline BMD at the femoral neck, and 3) advancing age. They also described that approximately $45 \%$ of fractures were attributable to these three risk factors, of which $13 \%$ was because of a high rate of bone loss and less than $32 \%$ was caused by osteoporosis. ${ }^{10}$ Among the elderly, each $0.12 \mathrm{~g} / \mathrm{cm}^{2}$ loss in femoral neck BMD is associated with a 3.1-fold increase in the risk of femoral neck fracture. ${ }^{9}$ The rate of femoral neck bone loss is an important risk factor for fracture. Approximately 300 cases of transient osteoporosis of the hip have so far been reported in the literature and pathological fracture occurred in three cases, at a mean of 8.3 weeks (5 to 12 ) from the onset of symptoms. ${ }^{4,6,11}$ The period of risk of pathological fractures is when there is a high rate of bone loss and when the BMD is at its lowest. For this reason nonweight-bearing is advisable.

No benefits in any form have been received or will be received from a commercial party related directly or indirectly to the subject of this article.

\section{References}

1. Curtiss PH, Kincaid WE. Transitory demineralization of the hip in pregnancy: a report of three cases. J Bone Joint Surg [Am] 1959;41-A:1327-33.

2. Schapira D. Transient osteoporosis of the hip. Semin Arthritis Rheum 1992;22:98-105.

3. Varenna M, Sinigaglia L, Binelli L, Beltrametti P, Gallazzi M. Transient osteoporosis of the hip: a densitometric study. Clin Rheumatol 1996;15:169-73.

4. Junk S, Ostrowski M, Kokoszczynski L. Transient osteoporosis of the hip in pregnancy complicated by femoral neck fracture: a case report. Acta Orthop Scand 1996;67:69-70

5. Varenna M, Zucchi F, Binelli L, et al. Intravenous pamidronate in the treatment of transient osteoporosis of the hip. Bone 2002;31:96-101.

6. Fokter SK, Vengust V. Displaced subcapital fracture of the hip in transient osteoporosis of pregnancy: a case report. Int Orthop 1997;21:201-3.

7. Carmona-Ortells L, Carvajal-Mendez I, Garcia-Alvaro JM, Alvaro-Gracia JM, Gonzalez-Alvaro I. Transient osteoporosis of the hip: successful response to deflazacort. Clin Exp Rheumatol 1995;13:653-5.

8. Montagna GL, Malesci D, Tirri R, Valentini G. Successful neridronate therapy in transient osteoporosis of the hip. Clin Rheumato/ 2005;24:67-9.

9. Arayssi TK, Tawbi HA, Usta IM, Horani MH. Calcitonin in the treatment of transient osteoporosis of the hip. Semin Arthritis Rheum 2003;32:388-97.

10. Nguyen TV, Center JR, Eisman JA. Femoral neck bone loss predicts fracture risk independent of baseline BMD. J Bone Miner Res 2005;20:1195-201.

11. Fingeroth RJ. Successful operative treatment of a displaced subcapital fracture of the hip in transient osteoporosis of pregnancy: a case report and review of the literature. $J$ Bone Joint Surg [Am] 1995;77-A:127-31. 